\title{
Genetic diversity of three surface protein genes in Plasmodium malariae from three Asian countries
}

\author{
Suttipat Srisutham ${ }^{1}$, Naowarat Saralamba ${ }^{1,2}$, Kanlaya Sriprawat ${ }^{3}$, Mayfong Mayxay ${ }^{4,5,6}$, Frank Smithuis ${ }^{7,8}$, \\ Francois Nosten ${ }^{3,8}$, Sasithon Pukrittayakamee ${ }^{2,9}$, Nicholas P. J. Day ${ }^{2,6}$, Arjen M. Dondorp ${ }^{2,6}$ \\ and Mallika Imwong ${ }^{1,2^{*}}$ (])
}

\begin{abstract}
Background: Genetic diversity of the three important antigenic proteins, namely thrombospondin-related anonymous protein (TRAP), apical membrane antigen 1 (AMA1), and 6-cysteine protein (P48/45), all of which are found in various developmental stages of Plasmodium parasites is crucial for targeted vaccine development. While studies related to the genetic diversity of these proteins are available for Plasmodium falciparum and Plasmodium vivax, barely enough information exists regarding Plasmodium malariae. The present study aims to demonstrate the genetic variations existing among these three genes in P. malariae by analysing their diversity at nucleotide and protein levels.

Methods: Three surface protein genes were isolated from 45 samples collected in Thailand $(N=33)$, Myanmar $(\mathrm{N}=8)$, and Lao PDR $(\mathrm{N}=4)$, using conventional polymerase chain reaction $(P C R)$ assay. Then, the PCR products were sequenced and analysed using BioEdit, MEGA6, and DnaSP programs.

Results: The average pairwise nucleotide diversities ( $\pi$ ) of $P$. malariae trap, ama1, and $p 48 / 45$ were $0.00169,0.00413$, and 0.00029 , respectively. The haplotype diversities $(\mathrm{Hd})$ of P. malariae trap, ama1, and $p 48 / 45$ were $0.919,0.946$, and 0.130 , respectively. Most of the nucleotide substitutions were non-synonymous, which indicated that the genetic variations of these genes were maintained by positive diversifying selection, thus, suggesting their role as a potential target of protective immune response. Amino acid substitutions of P. malariae TRAP, AMA1, and P48/45 could be categorized to 17, 20, and 2 unique amino-acid variants, respectively. For further vaccine development, carboxyl terminal of P48/45 would be a good candidate according to conserved amino acid at low genetic diversity $(\pi=0.2-0.3)$.

Conclusions: High mutational diversity was observed in P. malariae trap and ama1 as compared to $p 48 / 45$ in $P$. malariae samples isolated from Thailand, Myanmar, and Lao PDR. Taken together, these results suggest that P48/45 might be a good vaccine candidate against $P$. malariae infection because of its sufficiently low genetic diversity and highly conserved amino acids especially on the carboxyl end.
\end{abstract}

Keywords: Plasmodium malariae, TRAP, AMA1, P48/45

\section{Background}

Plasmodium malariae-related incidents are mostly observed in endemic areas [1] and have lower prevalence than Plasmodium falciparum or Plasmodium vivax

\footnotetext{
*Correspondence: noi@tropmedres.ac; mallika.imw@mahidol.ac.th

${ }^{2}$ Mahidol Oxford Tropical Medicine Research Unit, Faculty of Tropical

Medicine, Mahidol University, Bangkok, Thailand
}

Full list of author information is available at the end of the article infections [2]. Several studies involving sophisticated molecular detection methods have revealed more accurate picture of $P$. malariae infection indicating much higher prevalence of the disease than previously expected in many regions of the world [3-5]. Moreover, P. malariae infection often displays low parasitaemia and can occur as mixed infection involving $P$. falciparum or $P$. vivax [6]. Similarly, P. malariae infection can remain asymptomatic 
for longer period of time and lead to increase in mortality by causing chronic nephrotic syndrome [7-13]. Hence, $P$. malariae is an important species that should be included in malaria elimination programmes [5]. As an important part of malaria elimination programme, extensive studies are being carried out focusing on potential molecular targets for development of effective vaccines against all forms of human malaria. One of the main surface proteins used as targets for development of vaccines is merozoite surface protein 1 (MSP1), for which the genetic diversity of its corresponding $m s p 1$ gene has been previously analysed for $P$. malariae [14].

In this context, the investigation was focused on three important antigenic proteins found in $P$. malariae namely thrombospondin-related anonymous protein (PmTRAP), apical membrane antigen 1 (PmAMA1), and 6-cysteine protein $(\mathrm{PmP} 48 / 45)$ which are known to play significant role in developmental stages of the parasite. TRAP is a sporozoite surface protein essential for sporozoite gliding, motility, and hepatocyte invasion [15-17]. This protein was utilized as a pre-erythrocytic vaccine target against $P$. falciparum (ChAd63-MVA ME-TRAP), which could reduce burden of the liver parasite by $79-84 \%$ [18]. Likely, AMA1 is a merozoite surface protein that plays a significant role in red blood cell invasion [19-24]. Using AMA1 as blood stage malaria vaccine showed $64.3 \%$ efficacy against clinical $P$. falciparum malaria [25]. P48/45 is a gametocyte antigen required for fertilization of gametocytes [26]. Moreover, P48/45 has been regarded as a promising candidate for vaccine development by transmission blockage of gametocyte in malaria vector [27].

Genetic diversity arising through antigenic polymorphisms can cause change in expression of critical epitopes resulting in reduction or even complete loss of vaccine efficacy. The success of an effective long-term vaccine development is, therefore, depended on widescale assessment of the degree of genetic polymorphism among parasite populations in malaria endemic areas. Genetic diversity of trap, ama1, and p48/45 genes of $P$. falciparum and $P$. vivax have been analysed and reported by multiple independent studies [28-30]. Although genome sequence of $P$. malariae has been recently reported for its evolutionary history and phylogenetic classification [31], there barely exists a study entirely focusing on genetic diversity of TRAP, AMA1, and $\mathrm{P} 48 / 45$ genes of $P$. malariae. Thus, the present study is probably the first investigation aimed at isolation and analysis of genetic diversity of genes coding for these three surface protein in P. malariae. In particular, population genetic of $P$. malariae was analysed based on three genes namely, $P$. malariae thrombospondin-related anonymous protein gene (pmtrap), P. malariae apical membrane antigen 1 gene (pmama1), and $P$. malariae 6-cysteine protein gene (pmp48/45). In addition, the study also includes comparative analysis of three surface proteins across six human Plasmodium species. Findings from the current study might contribute to crucial information needed for further understanding and potential vaccine development against $P$. malariae infection.

\section{Methods \\ DNA samples}

DNA samples were extracted from total of 45 symptomatic $P$. malariae infected patients from Thailand $(\mathrm{N}=33)$, Myanmar $(\mathrm{N}=8)$, and Lao PDR $(\mathrm{N}=4)$ in 2002-2013 (Table 1). These DNA samples were purified from whole blood or dried blood spot on filter paper using QIAamp DNA Mini Kit (Qiagen, Germany) and then stored at $-80^{\circ} \mathrm{C}$. These samples were subjected to confirmative identification for Plasmodium species using polymerase chain reaction (PCR) based on 18 small-subunit ribosomal RNA $[32,33]$ to confirm the present of $P$. malariae.

\section{Amplification and sequencing of Plasmodium malariae trap, ama1, and $p 48 / 45$ genes}

Plasmodium malariae trap, ama1 and p48/45 genes were amplified using either nested or semi-nested PCR using

Table 1 DNA samples used in the study

\begin{tabular}{|c|c|c|c|c|c|c|c|}
\hline \multirow[t]{2}{*}{ Countries } & \multirow[t]{2}{*}{ Study sites } & \multirow[t]{2}{*}{ Collected year } & \multirow[t]{2}{*}{ No. of samples } & \multirow[t]{2}{*}{ Plasmodium species ${ }^{\mathrm{a}}$} & \multicolumn{3}{|l|}{ Results } \\
\hline & & & & & pmtrap & pmama1 & pmp48/45 \\
\hline \multirow[t]{2}{*}{ Thailand } & Tak & 2003-2012 & 22 & \multirow{2}{*}{$\begin{array}{l}P m(\mathrm{n}=25) \\
P m+P f(\mathrm{n}=3) \\
P m+P V(\mathrm{n}=3) \\
P m+P f+P V(\mathrm{n}=2)\end{array}$} & \multirow[t]{2}{*}{27} & \multirow[t]{2}{*}{32} & \multirow[t]{2}{*}{33} \\
\hline & Kanchanaburi & 2003-2013 & 11 & & & & \\
\hline Myanmar & Rakhine & 2009 & 8 & $\begin{array}{l}P m(n=7) \\
P m+P V(n=1)\end{array}$ & 5 & 7 & 7 \\
\hline \multirow[t]{2}{*}{ Lao PDR } & Savannakhet & 2010 & 4 & $P m(n=4)$ & 3 & 4 & 4 \\
\hline & Total & & 45 & & 35 & 43 & 44 \\
\hline
\end{tabular}

a Pm: Plasmodium malariae; $P m+P f:$ mixed $P$. malariae and $P$. falciparum; $P m+P v$ : mixed $P$. malariae and $P$. vivax; $P m+P f+P v:$ mixed $P$. malariae, $P$. falciparum, and $P$. vivax 
specifically designed primers with the optimized $\mathrm{MgCl}_{2}$ concentrations and annealing temperatures, which were validated previously (Additional file 1 ). PCR products were either cloned into pGEM T-easy vector (Promega, USA) or directly purified before DNA sequencing. The nucleotide sequences of $P$. malariae trap, ama1 and $p 48 / 45$ genes obtained in this study have been submitted to GenBank database under the accession numbers KY905247-KY905306.

\section{Sequence analysis}

The overall analysis procedure was divided into four major sections. Firstly, the nucleotide polymorphism of $P$. malariae trap, ama1 and $p 48 / 45$ genes were measured. Partial DNA sequences were aligned using BioEdit and then we calculated average pairwise nucleotide diversity $(\pi)$, the number of haplotypes $(\mathrm{H})$, and haplotype diversity (Hd) using DnaSP v5 [34]. The nucleotide diversity was also plotted using a sliding method with a window length of 60 base pairs (bp) and a step size of $3 \mathrm{bp}$ using DnaSP v5. Moreover, partial nucleotide sequences were aligned with reference sequences of $P$. malariae trap, ama1, and $p 48 / 45$ isolated from Uganda, accession numbers LT594633.1, LT594630.1, and LT594500.1, respectively. In addition, we also aligned the nucleotide sequences of $P$. malariae trap, ama1, and p48/45 with sequences isolated from Malaysia, Indonesia, and Guinea, accession numbers ERS1110317, ERS1110321, and ERS567899, respectively [31].

Secondly, the amino acid substitutions and haplotype diversity of $P$. malariae TRAP, AMA1, and P48/45 were identified. Partial nucleotide sequences of $P$. malariae trap, ama1, and p48/45 were translated to deduced amino acid sequences using BioEdit and then aligned with references amino acid sequences of $P$. malariae TRAP, AMA1, and P48/45, accession numbers SCO93694.1, SCN12851.1, and SBT79956.1, respectively. The functional domains of these three proteins based on their reference amino acid sequences were predicted using InterPro [35] (Additional file 2).

Thirdly, P. malariae population was characterized based on three protein genes, the codon-based test of purifying selection was analysed using MEGA6 program [36]. The mean number of synonymous mutations per synonymous site $(\mathrm{dS})$ and non-synonymous substitutions per non-synonymous site $(\mathrm{dN})$ within each isolate were calculated using the Nei and Gojobori method [37] with the Jukes and Cantor correction. The statistical differences between $\mathrm{dN}$ and $\mathrm{dS}$ of the three genes were tested using Z-test of selection. In addition, neutral model was utilized to determine whether polymorphism took place at higher or lower frequencies than expected. Three population genetic tests of neutrality (Tajima's D, Fu and Li'
$\mathrm{D}^{*}$ and $\mathrm{Fu}$ and $\mathrm{Li}^{\prime} \mathrm{F}^{*}$ tests) $[38,39]$ were applied to the $P$. malariae trap, ama 1 and $p 48 / 45$ gene sequences.

In addition, to provide additional information that may further contribute to current knowledge of genetic polymorphism existing among different human Plasmodium species, the nucleotide diversity and amino acid homology of TRAP, AMA1, and P48/45 surface proteins among six Plasmodium species were analysed using reference nucleotide and amino acid sequences obtained from NCBI databases (Additional file 3). Maximum likelihood trees of six human malaria parasites were constructed using MEGA6 program based on amino acid sequences of TRAP, AMA1, and P48/45 [36] to show the phylogeny among six Plasmodium species.

\section{Results}

\section{Comparative analysis of trap, ama1 and p48/45 gene within six human Plasmodium species}

To provide information of diversity in trap, ama1, and p48/45 across all human malaria species, the reference sequences for trap, ama1, and p48/45 from all Plasmodium species were retrieved from database and analysed. The genetic variation was measured using a sliding method plot with a window length of $60 \mathrm{bp}$, a step size of $3 \mathrm{bp}$, and exclusion of sites with gaps. Results revealed overall $\pi$ value for $p 48 / 45$ in range $0.19000-0.51000$. However, low diversity was observed after $1 \mathrm{~kb}$ length with approximate $\pi$ values in the range $0.2-0.3$ (Fig. 1). The $\pi$ diversity was also measured for trap and ama1 with the overall observed range of 0.20111-0.55333 and $0.17111-0.45333$, respectively. The diversity was observed with fluctuated peak along the length of both trap and ama1 (Fig. 1). Multiple sequence alignment of those three corresponding surface proteins of all human Plasmodium species were determined. Overall sequence homology for TRAP, AMA1, and P48/45 varied from 36.44 to $89.38,54.02-96.08$, and $55.92-96.51 \%$,

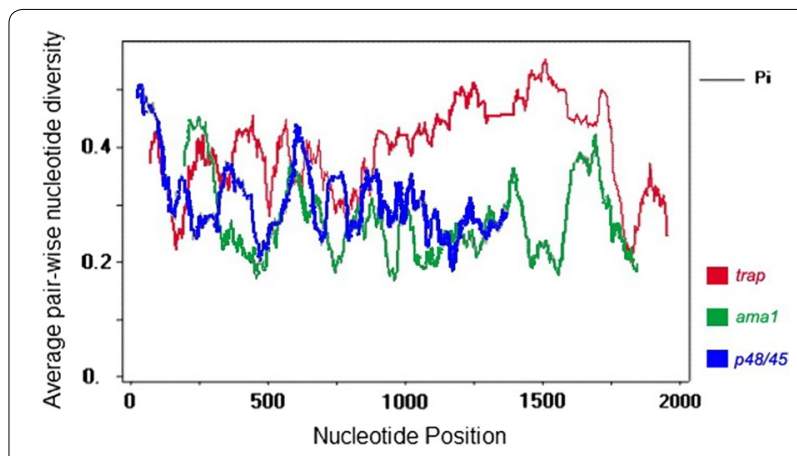

Fig. 1 Sliding window plots of the average pair-wise nucleotide diversity $(\pi)$. The nucleotide diversity of six Plasmodium species trap (red), ama 1 (green) and $p 48 / 45$ (blue) were plotted by a sliding window with a window size of $60 \mathrm{bp}$, a step size of $3 \mathrm{bp}$, and excluding sites with gaps 
respectively (Additional file 4). Among human malaria parasites, $P$. malariae TRAP, AMA1, and P48/45 high homology with Plasmodium ovale wallikeri TRAP (47.47\%), AMA1 (71.40\%) and P48/45 (63.34\%), respectively. Additional comparison of $P$. malariae TRAP, AMA1, and P48/45 with $P$. falciparum and with $P$. vivax showed that the homology of $P$. malariae and $P$. vivax TRAP (45.97\%), AMA1 (68.68\%), and P48/45 (61.40\%) were higher than the homology of $P$. malariae and $P$. falciparum TRAP (36.44\%), AMA1 (56.77\%), and P48/45 (58.90\%), respectively (Additional file 4). Conserved amino acids were found partially along those three gene sequences. The highest level of homology across all Plasmodium species was observed in P48/45 with the highly conserved residues located near the carboxyl terminal. The maximum likelihood tree showed that $P$. falciparum TRAP, AMA1, and P48/45 were less related with other Plasmodium species. In addition, the phylogeny shows recent common ancestry of $P$. malariae and $P$. ovale based on TRAP and P48/45 (Additional file 5).

\section{Nucleotide polymorphism of Plasmodium malariae trap, ama1, and $p 48 / 45$}

Using PCR the 35,43 , and 44 isolates of $P$. malariae were successfully amplified for trap, ama1 and $p 48 / 45$ genes (Table 1). The 35 isolates of $P$. malariae trap were amplified corresponding to nucleotide positions 172-1824 of $P$. malariae reference sequence accession number LT594633.1. The fragment size of pmtrap varied from 1653 to $1713 \mathrm{bp}$, in which the variation resulted from difference of number of repeat units. There were three patterns of 12 nucleotides repeat units, including CCAG AGGATAGA, CCAGAGAATAGA, and CCAGAGAATA GT. Among those three patterns, the most frequent repeat was CCAGAGGATAGA which accounted for $75-92 \%$ of all observed repeats. Excluding repeat regions, there were seven polymorphic nucleotide sites found among 35 samples with an average $\pi$ value of 0.00169 . A sliding method plot with a window length of $60 \mathrm{bp}$ and a step size of $3 \mathrm{bp}$ using DnaSP v5 revealed a $\pi$ value in range 0.00001-0.01289 (Fig. 2). Nucleotide diversity could be categorized to 16 distinguish haplotypes with an estimated Hd of 0.919 (Table 2).

Partial nucleotide sequences of amal gene were obtained from 43 P. malariae samples, spanning nucleotide positions 52-1632 of $P$. malariae reference sequence accession number LT594630.1. In all of the 43 P. malariae isolates ama1 sequences had the same amplification size of $1581 \mathrm{bp}$. There were 17 polymorphic nucleotide sites found among 43 samples with an average $\pi$ value of 0.00420 . Use of sliding method plot with a window length of $60 \mathrm{bp}$ and a step size of $3 \mathrm{bp}$ revealed a $\pi$ value in range 0.00001-0.03230 (Fig. 2). Nucleotide diversity could be

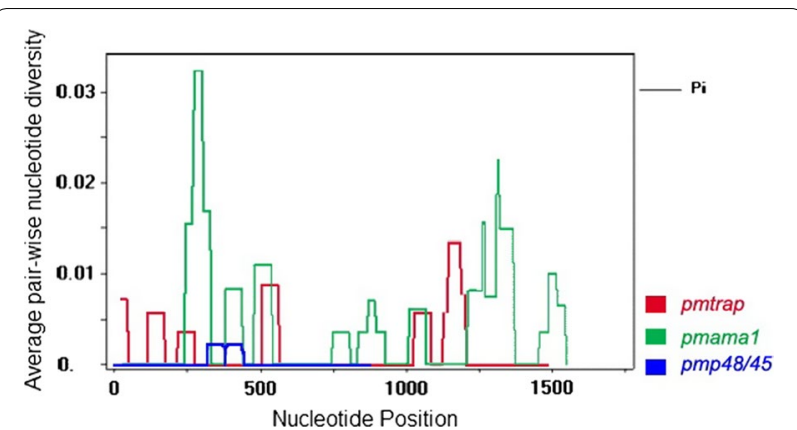

Fig. 2 Sliding window plots of the average pair-wise nucleotide diversity ( $\pi$ ). The nucleotide diversity of P. malariae trap (red), pmama 1 (green) and pmp48/45 (blue) isolated from Thailand, Myanmar, and Lao PDR were plotted by a sliding window with a window size of $60 \mathrm{bp}$ and a step size of $3 \mathrm{bp}$

categorized to 24 distinct haplotypes with an $\mathrm{Hd}$ value of 0.948 (Table 2).

The partial sequences of $p 48 / 45$ were successfully amplified from $44 P$. malariae samples, covering nucleotide positions 244-1152 of $P$. malariae reference strain, PMUG01 accession number LT594500.1. Those 44 samples of $P$. malariae $p 48 / 45$ genes were isolated each having the same size of $909 \mathrm{bp}$. There were two polymorphic nucleotide sites having an average $\pi$ value of 0.00028 . Use of sliding method plot with a window length of $60 \mathrm{bp}$ and a step size of 3 bp revealed a $\pi$ value in range 0.000010.00217 (Fig. 2). Nucleotide sequence alignment analysis suggested presence of only two distinct haplotypes with an estimated Hd value of 0.127 (Table 2).

Nucleotide diversity of $P$. malariae trap, ama1, and p48/45 obtained from this study were compared with the nucleotide diversity calculated for five $P$. malariae samples and two $P$. malariae-like (Chimpanzee infecting) parasites obtained from previous study [31]. The study result is in agreement with the previously published report of similar pattern of highest nucleotide diversity found in P. malariae ama1 as compared to trap and $p 48 / 45$. Moreover, nucleotide diversity and haplotype diversity of $P$. malariae trap, ama1 and $p 48 / 45$ isolated during this study were compared with $P$. falciparum and $P$. vivax from previous reports $[28,30,40]$ (Table 2). It was found that $P$. malariae trap, ama1 and $p 48 / 45$ showed lower nucleotide diversity than $P$. falciparum and P. vivax $(\mathrm{P}<0.05)$.

\section{Amino acid substitutions and variations of Plasmodium malariae TRAP, AMA1 and P48/45}

The isolated nucleotide sequences of trap, amal, and $p 48 / 45$ from all $P$. malariae samples were translated to deduce their corresponding amino acids and analysed. The trap, ama1, and p48/45 of P. malariae were intron 
Table 2 Average nucleotide diversity and haplotype diversity of three surface genes of $P$. malariae, $P$. falciparum, and $P$. vivax

\begin{tabular}{|c|c|c|c|c|c|c|c|}
\hline \multirow[t]{2}{*}{ Gene } & \multicolumn{3}{|c|}{ Haplotype diversity (n) } & \multicolumn{3}{|c|}{ Average nucleotide diversity $\pi\left(\times 10^{-3}\right)(\mathrm{n})$} & \multirow{2}{*}{$\begin{array}{l}\text { Average nucleotide diversity }(\pi) \\
\text { P. malariae and P. malariae-liked }\end{array}$} \\
\hline & P. falciparum & P. vivax & P. malariae & P. falciparum & P. vivax & P. malariae & \\
\hline trap & $0.990(29)^{\mathrm{a}}$ & $0.960(63)^{b}$ & $0.919(35)$ & $6.120(29)^{\mathrm{a}}$ & $7.070(63)^{b}$ & $1.690(35)$ & $0.64^{d}$ \\
\hline amal & $0.940(55)^{\mathrm{a}}$ & $0.899(235)^{b}$ & $0.948(43)$ & $25.500(55)^{\mathrm{a}}$ & $14.880(235)^{b}$ & $4.200(43)$ & $3.58^{d}$ \\
\hline$p 48 / 45$ & $0.200(10)^{\mathrm{a}}$ & $0.484(18)^{c}$ & $0.127(44)$ & $0.600(10)^{a}$ & $1.160(18)^{c}$ & $0.280(44)$ & $0.28^{d}$ \\
\hline
\end{tabular}

a $[28],{ }^{b}[29],{ }^{c}[30],{ }^{d}[31]$

less genes. The translated PmTRAP was found to be located on amino acid position 58-608 of P. malariae reference strain PMUG01 accession number SCO93694.1. The tandem repeat of 12 nucleotide unit corresponded to the three tetrapeptide unit including PEDR, PENR, and PENS, located within TSP1 domain. Number of total repeat unit among those 35 samples of $P$. malariae isolates varied from 9 to 17 repeats. The major repeat unit was PEDR which could be found from 8 to 14 units in all isolates. All of the isolates contained only one unit of PENR, except for none in one isolate from Myanmar. The repeat unit PENS also presented for 1-2 units in most $P$. malariae samples, whereas only two isolates of $P$. malariae collected in Thailand had none. Analysis of PmTRAP in non-repeat region showed seven polymorphic sites that resulted from non-synonymous mutations. Those seven polymorphic sites were $62(\mathrm{~S} / \mathrm{F}), 105(\mathrm{I} / \mathrm{V}), 139$ (F/L), 236 (I, T), $454(\mathrm{G}, \mathrm{R}), 488(\mathrm{H}, \mathrm{R})$, and $494(\mathrm{D}, \mathrm{G})$. Alignment of the 35 PmTRAP isolates revealed 17 variant types, of which the variant 9 (SILIRHD) was seen in 8 out of 35 isolates, thus, making it the most frequently observed variant type among the samples (Figs. 3, 6a, Additional file 6).

The translated PmAMA1 was corresponding to amino acid positions $18-544$ of $P$. malariae reference strain, PMUG01, accession number SCN12851.1. There were 527 residues of PmAMA1 from 43 isolates of $P$. malariae for analysis. Sequence alignment of-PmAMA1 from those 43 isolates revealed 18 polymorphic sites at the nucleotide level, which in turn consisted of 13 non-synonymous sites and one synonymous position. The pattern of PmAMA1 polymorphisms observed in 43 P. malariae isolates could be classified into 20 variant types. Variant type 5 was found with the highest frequency in 10 out of 43 isolates (Figs. 4, 6b, Additional file 6). Most of the samples expressing variant type 5 were from Thailand $(\mathrm{N}=9)$.

The P48/45 were translated from 44 P. malariae samples, spanning amino acid residue 82-384 of $P$. malariae reference strain, PMUG01, accession number SBT79956.1. Total of 303 residues from 44 samples of
PmP48/45 were analysed and results showed two nonsynonymous sites at 202 (I, K) and 203 (F, L) (Figs. 5, $6 \mathrm{c}$, Additional file 6). There were only two variant types found among those 44 P. malariae isolates. There were 41 samples found in variant 1 while only 3 samples of P. malariae exhibited variant type 2 (Fig. 5). Previous report of PmP48/45 sequences analysis from Malaysia, Indonesia and Guinea [31] contained the same haplotype pattern as variant type 1, while the isolate from Uganda [31] had the same haplotype as variant type 2 .

\section{Genetic population testing of $P$. malariae trap, ama1, and $p 48 / 45$}

Population genetics of $P$. malariae was analysed based on trap, ama1, and $p 48 / 45$ gene polymorphism. The codonbased test of purifying selection was analysed using MEGA6 program. The average $\mathrm{dS}$ and $\mathrm{dN}$ values within each isolate were calculated using $\mathrm{Nei}$ and Gojobori method [37] with Jukes and Cantor correction. Statistical differences between $\mathrm{dN}$ and $\mathrm{dS}$ were tested with the Z-test of selection using the MEGA6 program. Results indicated that there was positive selection or diversifying selection in P. malariae trap, ama1, and $p 48 / 45$ population (Prob $=1.000, \mathrm{dS}-\mathrm{dN}=-2.509$ (trap), -1.243 (ama1), and - 1.479 (p48/45). Besides, these three population genetic tests, the additional test for neutrality (Tajima's D, Fu and Li' D* and Fu and Li' F* tests) [38, 39] was applied to the pmtrap, pmama1 and pmp $48 / 45$ sequences to determine whether polymorphism takes place at higher or lower frequencies than expected under a neutral model. Tajima's $\mathrm{D}, \mathrm{Fu}$ and $\mathrm{Li} \mathrm{D}^{*}$, and $\mathrm{Fu}$ and $\mathrm{Li}$ $\mathrm{F}^{*}$ tests using DnaSP v5 and MEGA6 were performed for pmtrap, pmama1 and pmp48/45. The results from Tajima's $\mathrm{D}$ value was 1.83835 for pmama1 $(\mathrm{P}<0.10)$, which was similar to $\mathrm{Fu}$ and $\mathrm{Li}^{\prime} \mathrm{F}^{*}$ test value $1.71236(\mathrm{P}<0.05)$ (Table 3). Significantly positive values for these statistics reflect an excess of intermediate-frequency alleles, which can result from population bottlenecks, structures or balancing selections. The Tajima's $\mathrm{D}, \mathrm{Fu}$ and $\mathrm{Li}^{\prime} \mathrm{D}^{*}$ and $\mathrm{Fu}$ and $\mathrm{Li}^{\prime} \mathrm{F}^{*}$ tests of pmtrap and pmp48/45 were not statistically significant $(P>0.10)$. Moreover, Fu's Fs statistic 


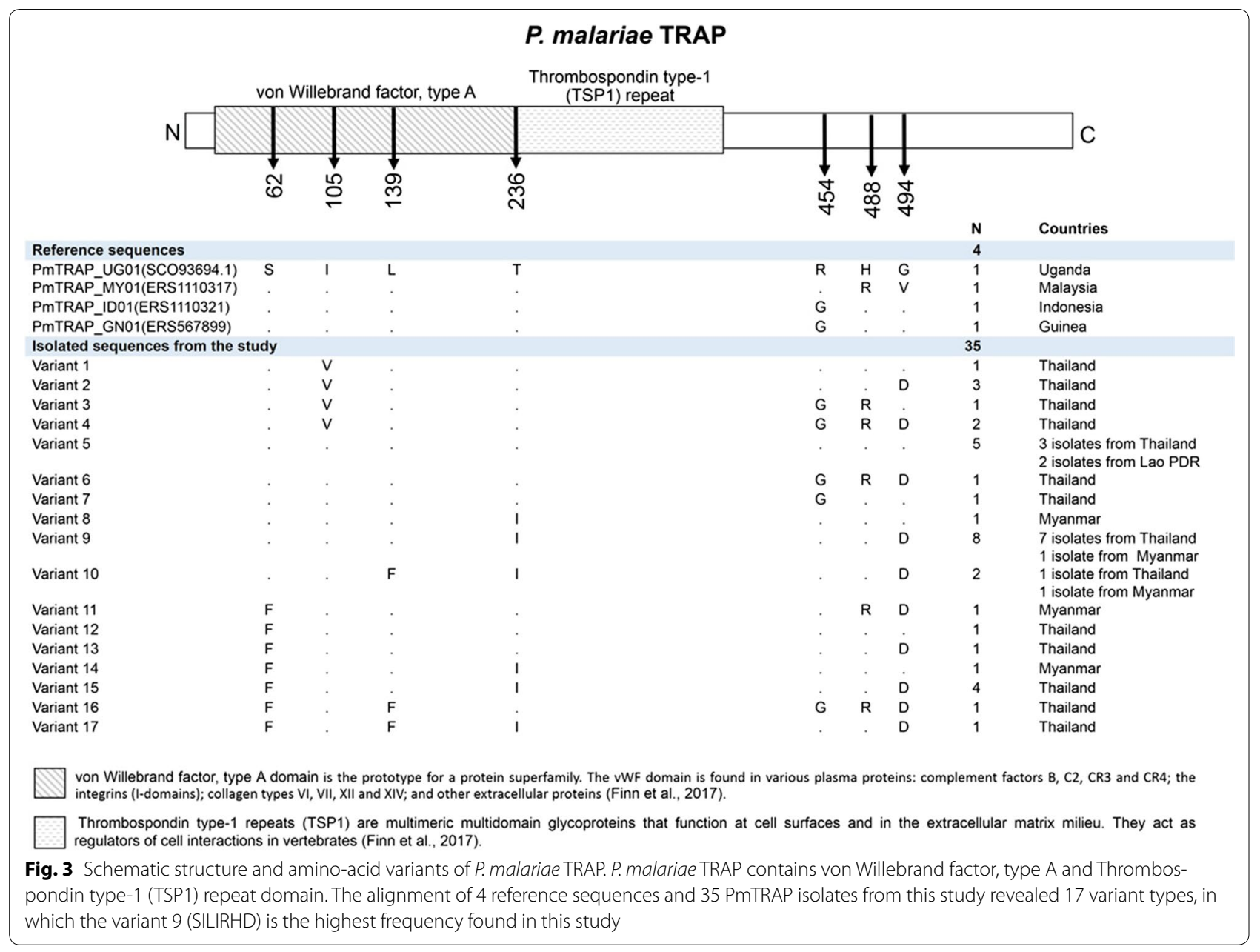

was negative for pmtrap and pmamal which indicated population expansion of trap and ama1 of P. malariae in the study samples.

\section{Discussion}

The development of modern high-throughput ultrasensitive diagnostic assays have demonstrated that $P$. malariae is more widely circulated in malaria endemic regions than previously acknowledged. Hence, this makes study of $P$. malariae essential to be included in malaria elimination programme [3-5]. Effective vaccine protecting all human malarias should be developed as part of malaria elimination programme. The lack of adequate information regarding genetic polymorphism and its underlying mechanism of the genes coding for critical surface proteins such as TRAP, AMA1, and P48/45 in P. malariae has limited limits current understanding of the parasite.

Genetic analysis for nucleotide and haplotype diversity suggests that pmp48/45 has the lowest diversity among all of the studied genes. There were only two unique amino-acid variants found in PmP48/45 compare to 17 and 20 amino-acid variants observed in PmTRAP and PmAMA1, respectively. Moreover, results from comparative analysis of six human Plasmodium species showed lowest diversity of $p 48 / 45$ among the parasites. In accordance to these finding, highly conserved expression of $p 48 / 45$ have been previously reported in $P$. falciparum [28] and P. vivax [30] isolates which might be related to the critical function of this protein for transmission and oocyst development of the parasite [26]. Low variation observed in $p 48 / 45$ nucleotide sequence coding for $\mathrm{P} 48 / 45$ protein and its relatively conserved amino acid residues may be linked to the gametocyte stage where it its mainly expressed. Interestingly, this protein has been previously investigated in other Plasmodium species for development of potential malaria vaccine targeting transmission stage of the parasite [27].

Noticeable levels of nucleotide polymorphism observed within $P$. malariae ama1 suggests that $P$. malariae may have substantial potency to exhibit genetic variation or acquire mutation on pmamal protein expression. In particular, most of the polymorphism were found 


\section{P. malariae AMA1}

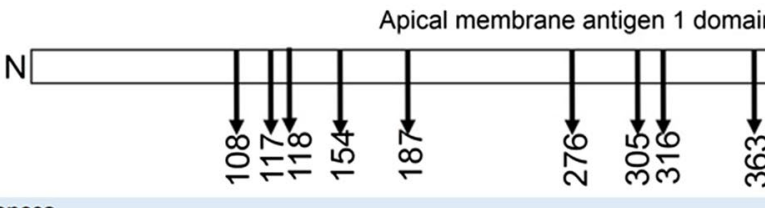

Reference sequences

PmAMA1 UG01(SCN128511)

PMAMA1_MYO(ERS1110317)

PmAMA1_ID01(ERS1110321)

PmAMA1_GN01(ERS567899)

Isolated sequences from the study

Variant 1

Variant 2

Variant 3

Variant 4

\begin{tabular}{|c|c|c|c|}
\hline & $\mathrm{N}$ & $\mathrm{R}$ & $\mathrm{N}$ \\
\hline G & $Y$ & . & $\mathrm{K}$ \\
\hline$\hat{N}$ & $\cdot$ & 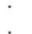 & K \\
\hline $\mathrm{N}$ & . & . & K \\
\hline $\mathrm{N}$ & . & . & $\mathrm{K}$ \\
\hline$G$ & $Y$ & $\mathrm{~K}$ & $\mathrm{~K}$ \\
\hline$G$ & $Y$ & $\mathrm{~K}$ & $\mathrm{~K}$ \\
\hline $\mathrm{N}$ & . & . & $\mathrm{K}$ \\
\hline $\mathrm{N}$ & . & . & $\mathrm{K}$ \\
\hline & - & . & $\mathrm{K}$ \\
\hline $\mathrm{N}$ & $Y$ & . & $\mathrm{K}$ \\
\hline G & $Y$ & . & $\mathrm{K}$ \\
\hline G & $Y$ & . & $\mathrm{K}$ \\
\hline & $Y$ & & $\mathrm{~K}$ \\
\hline
\end{tabular}

त लू ल

Variant 5

Variant 6

Variant 7

Variant 8

Variant 9

Variant 10

Variant 11

Variant 12

Variant 13

$\begin{array}{ccccc}\cdot & \cdot & \mathrm{K} & \cdot & \\ \mathrm{N} & \cdot & \mathrm{K} & \cdot & \\ \mathrm{N} & \cdot & \cdot & \cdot & \mathrm{N} \\ \mathrm{N} & \cdot & \cdot & \cdot & \mathrm{N} \\ \mathrm{N} & \cdot & \cdot & \cdot & \cdot \\ \mathrm{N} & \cdot & \cdot & \cdot & \cdot \\ \cdot & \cdot & \cdot & \cdot & \cdot \\ & & \cdot & \end{array}$

Variant 14

Variant 15

Variant 16

Variant 17

Variant 18

Variant 19

Variant 20

D $K \mathrm{P} \quad \mathrm{L}$

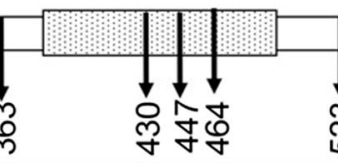

N Countries

$\mathrm{N}$

$\begin{array}{llllll}N & \text { L } & \text { V } & \text { D } & 1 & \text { Uganda } \\ \text { K } & . & . & & 1 & \text { Malaysia }\end{array}$

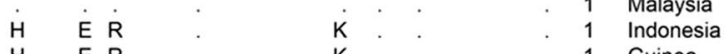

H E R

$R$

1 Guinea

A

Apical membrane antigen 1 domain is the protein domain that contains a hydrophobic domain typical of an integral membrane protein. The antigen is designated apical membrane antigen 1 (AMA-1) by virtue of appearing to be located in the apical complex. AMA-1 appears to be transported to the merozoite surface close to the time of schizont rupture. (Finn et al., 2017).

Fig. 4 Schematic structure and amino-acid variants of P. malariae AMA1. P. malariae AMA1 contains apical membrane antigen 1 domain. The alignment of 4 reference sequences and 43 PmAMA1 isolates from this study revealed 20 variant types, in which the variant 5 (GYKKNDKPLKVIN) is the highest frequency found in this study

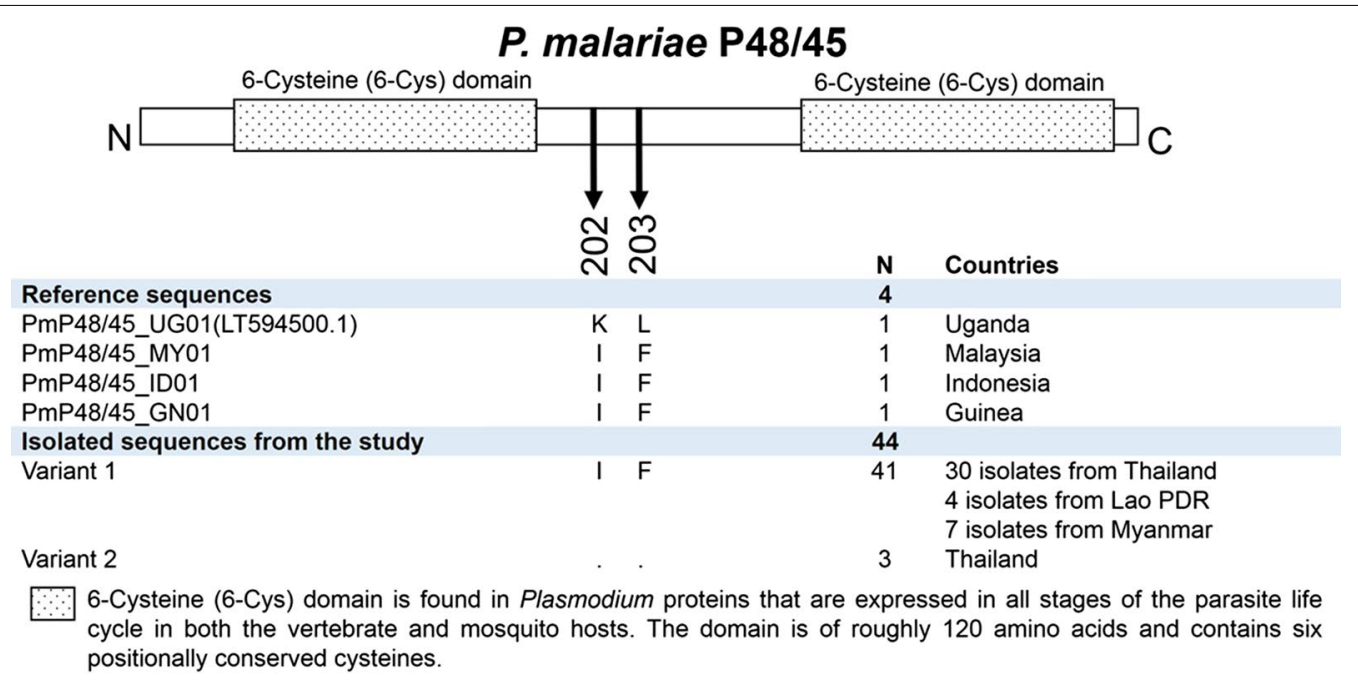

Fig. 5 Schematic structure and amino-acid haplotypes of P. malariae P48/45. P. malariae P48/45 contains two regions of 6-cysteine domain. The alignment of 4 reference sequences and $44 \mathrm{PmP} 48 / 45$ isolates from this study revealed 2 variant types, in which the variant 1 (IF) is the highest frequency found in this study 

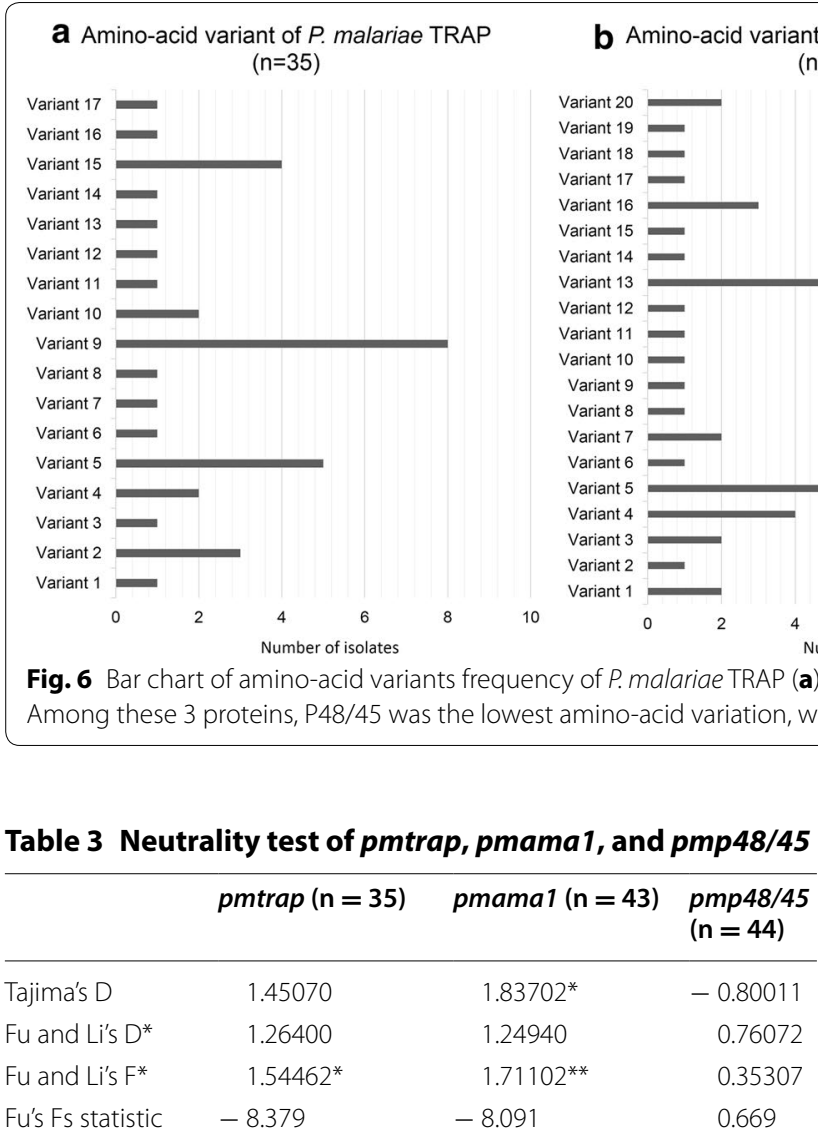

${ }^{*} 0.05<\mathrm{P}<0.10,{ }^{* *} \mathrm{P}<0.05$

at N-terminal position indicating possibility of directional mutation of pmamal gene favoring $\mathrm{N}$-terminallinked functions of the protein. The genetic diversity observed in $P$. malariae trap was comparatively lower than pmama1. These results are in agreement with the previously published report of similar pattern of high nucleotide diversity found in P. falciparum ama1 as compared to P. falciparum trap in regions of America, Asia Pacific, and Africa [28]. This noticeable level of genetic polymorphism observed in ama1 of six Plasmodium species might be related to the stage of parasite infection and functional aspect of the protein. The Plasmodium expresses AMA1 during merozoite stage of the parasite where it has to actively evade host immunity. Thus, acquiring genetic diversity in such crucial functional proteins can assist avoidance of host detection and subsequent neutralization of the parasite. Since AMA1 and TRAP both are expressed during active infection stages of Plasmodium, the evolutionary requirement level of genetic polymorphism might have been considerably higher for these genes as compared to P48/45 which is mainly expressed in gametocytes. In addition, within the $P$. malariae trap, the twelve nucleotides repeat was frequently observed for 9-17 times in the samples indicating possibility of this repeat to be used as genotyping marker.

The pmtrap and pmamal genes analysed during this study exhibited relatively more nucleotide diversity than pmp48/45. Both of these genes and their expression proteins have been previously targeted for development of pre-erythrocytic and blood stage vaccines against $P$. falciparum based on their immunogenic and conserved regions $[18,25]$. These findings indicated presence of conserved regions in P. malariae TRAP and AMA1, which might be useful as potential vaccine target against the parasite. These regions containing largest amount of conserved amino acid sequences for PmTRAP $(\mathrm{N}=35)$ and PmAMA1 $(\mathrm{N}=43)$ were found to be located in positions $237-453$ and $18-107$, respectively. In addition, the nucleotide diversities observed in pmtrap and pmama1 during our observation were significantly lower compared to the levels previously reported in $P$. falciparum and $P$. vivax $[28,40]$. Thus, these results collectively suggests the possibility to develop malaria vaccine against $P$. malariae targeting conserved regions of PMTRAP and PmAMA1.

The ability of parasite to evolve and survive is greatly influenced by its ability to acquire genetic mutations to withstand host immunity and environmental stress [28, 40]. The results indicated higher occurrence of non-synonymous mutation in P. malariae trap, ama1, and $p 48 / 45$ genes suggesting positive or diversifying selection. In addition, the results from $\mathrm{Z}$-test indicates that the polymorphisms in these genes were most probably achieved through positive diversifying selection. Such mechanisms have been reported to be favourable for the parasite to 
evade targeted host immune responses [28, 30, 40]. However, future study involving large number of samples from diverse geographical locations can help create clearer picture of the genetic polymorphism in P. malariae and their possible implications in malaria research.

In addition, the $P$. malariae trap, amal and $p 48 / 45$ showed lower nucleotide diversity compared to $P$. falciparum and $P$. vivax, which might have been linked to the lower transmission rate of $P$. malariae in these geographic regions [41] and the limited number of $P$. malariae samples analysed. Nonetheless, these findings were similar to previously published data that reported low level of sequence diversity of $m s p 1$ gene in $P$. malariae parasite as opposed to other Plasmodium species [14].

\section{Conclusions}

In summary, present investigation is the first reported study revealing genetic diversity of three surface protein coding genes namely trap, ama1, and $p 48 / 45$ in $P$. malariae. Results of this study inevitability contributes to the malaria research and may potentially be a valuable resource for further vaccine development or genotyping marker.

\section{Additional files}

Additional file 1. Specific primers and PCR conditions for isolation of 3 surface protein genes.

Additional file 2. Domain prediction of P. malariae TRAP, AMA1, and P48/45 using InterPro.

Additional file 3. Accession numbers of amino acid sequences and gene IDs of six Plasmodium TRAP, AMA1, and P48/45 obtained from database.

Additional file 4. Amino acid identity of non-cytoplasmic region TRAP, $A M A 1$, and P48/45 within six Plasmodium species.

Additional file 5. Maximum likelihood trees of six human malaria parasites based on amino acid sequences of TRAP, AMA1, and P48/45.

Additional file 6. PmTRAP1, PmAMA1, and PmP48/45 variant frequencies in Thailand, Myanmar, and Lao PDR.

\section{Abbreviations}

TRAP: thrombospondin-related anonymous protein; AMA1: apical membrane antigen 1 protein; $\mathrm{P} 48 / 45$ : 6-cysteine protein; MSP1: merozoite surface protein 1; Hd: haplotype diversities; $\pi$ : average pairwise nucleotide diversities.

\section{Authors' contributions}

SS, NS, and MI contributed to study design. KS, MM, FS, FN, SP, and ND collected samples. SS and NS undertook laboratory work. SS, NS, AD, and MI analysed data. SS, NS, and MI drafted the manuscript. All authors read and approved the final manuscript.

\footnotetext{
Author details

${ }^{1}$ Department of Molecular Tropical Medicine and Genetics, Faculty of Tropical Medicine, Mahidol University, Bangkok, Thailand. ${ }^{2}$ Mahidol Oxford Tropical Medicine Research Unit, Faculty of Tropical Medicine, Mahidol University, Bangkok, Thailand. ${ }^{3}$ Shoklo Malaria Research Unit, Mahidol-Oxford Tropical Medicine Research Unit, Mae Sot, Thailand. ${ }^{4}$ Lao-Oxford-Mahosot Hospital-Wellcome Trust Research Unit (LOMWRU), Microbiology Laboratory, Mahosot Hospital, Vientiane, Lao People's Democratic Republic. ${ }^{5}$ Faculty
}

of Postgraduate Studies, University of Health Sciences, Vientiane, Lao People's Democratic Republic. ${ }^{6}$ Centre for Tropical Medicine and Global Health, Churchill Hospital, University of Oxford, Oxford, UK. ${ }^{7}$ Medical Action Myanmar, Yangon, Myanmar. ${ }^{8}$ Centre for Tropical Medicine, Nuffield Department of Clinical Medicine, University of Oxford, Oxford OX3 7LF, UK. ${ }^{9}$ Department of Clinical Tropical Medicine, Faculty of Tropical Medicine, Mahidol University, Bangkok, Thailand.

\section{Acknowledgements}

We thank Prof. Paul N. Newton for the samples collection from Lao PDR and the healthcare workers involved in sample collection across all study sites. We also thank Dr. Kemavit Bhangananda for his help.

\section{Competing interests}

The authors declare that they have no competing interests.

\section{Availability of data and materials}

The nucleotide sequences of P. malariae trap, ama 1 and $p 48 / 45$ genes obtained from this study have been submitted in GenBank database under the Accession Numbers KY905247-KY905306.

\section{Consent for publication \\ Not applicable.}

\section{Ethics approval and consent to participate}

The protocol of this study was reviewed and approved by the ethical review board of Faculty of Tropical Medicine, Mahidol University, Thailand (MUTM 2015-032-01).

\section{Funding}

This study was financed in part by Mahidol University, Thailand, and was part of the Wellcome Trust Mahidol University-Oxford Tropical Medicine Research Programme supported by the Wellcome Trust of Great Britain. NS is supported by Dean's Research Fund, 2012, Faculty of Tropical Medicine, Mahidol University. SS is supported by the Royal Golden Jubilee Ph.D. Programme, the Thailand Research Fund (PHD/0232/2553).

\section{Publisher's Note}

Springer Nature remains neutral with regard to jurisdictional claims in published maps and institutional affiliations.

Received: 7 November 2017 Accepted: 8 January 2018

Published online: 11 January 2018

\section{References}

1. Collins WE, Jeffery GM. Plasmodium malariae: parasite and disease. Clin Microbiol Rev. 2007;20:579-92.

2. Bruce MC, Macheso A, Galinski MR, Barnwell JW. Characterization and application of multiple genetic markers for Plasmodium malariae. Parasitology. 2007;134:637-50.

3. Camargo-Ayala PA, Cubides JR, Nino CH, Camargo M, Rodriguez-Celis CA, Quinones T, et al. High Plasmodium malariae prevalence in an endemic area of the Colombian Amazon region. PLoS ONE. 2016;11:e0159968.

4. Cao Y, Wang W, Liu Y, Cotter C, Zhou H, Zhu G, et al. The increasing importance of Plasmodium ovale and Plasmodium malariae in a malaria elimination setting: an observational study of imported cases in Jiangsu Province, China, 2011-2014. Malar J. 2016;15:459.

5. Lo E, Nguyen K, Nguyen J, Hemming-Schroeder E, Xu J, Etemesi H, et al. Plasmodium malariae prevalence and csp gene diversity, Kenya, 2014 and 2015. Emerg Infect Dis. 2017;23:601-10.

6. Lalremruata A, Jeyaraj S, Engleitner T, Joanny F, Lang A, Belard S, et al. Species and genotype diversity of Plasmodium in malaria patients from Gabon analysed by next generation sequencing. Malar J. 2017;16:398.

7. Wang ZY, Zhang YG, Jiang L, Li M, Zhu M, Cal L. Laboratory analysis and diagnosis of one transfusion-transmitted quartan malaria case in Shanghai City. Zhongguo Xue Xi Chong Bing Fang Zhi Za Zhi. 2015;27:362-6 (in Chinese) 
8. Brouwer EE, van Hellemond JJ, van Genderen PJ, Slot E, van Lieshout $L$, Visser $L G$, et al. A case report of transfusion-transmitted Plasmodium malariae from an asymptomatic non-immune traveller. Malar J. 2013;12:439.

9. Scuracchio P, Vieira SD, Dourado DA, Bueno LM, Colella R, Ramos-Sanchez EM, et al. Transfusion-transmitted malaria: case report of asymptomatic donor harboring Plasmodium malariae. Rev Inst Med Trop Sao Paulo. 2011;53:55-9.

10. Langford S, Douglas NM, Lampah DA, Simpson JA, Kenangalem E, Sugiarto $P$, et al. Plasmodium malariae infection associated with a high burden of anemia: a hospital-based surveillance study. PLoS Negl Trop Dis. 2015;9:e0004195.

11. Roman DN, Rosalie NN, Kumar A, Luther KM, Singh V, Albert MS. Asymptomatic Plasmodium malariae infections in children from suburban areas of Yaoundé, Cameroon. Parasitol Int. 2018:67:29-33.

12. Eiam-Ong S. Malarial nephropathy. Semin Nephrol. 2003;23:21-33.

13. Hedelius R, Fletcher JJ, Glass WF 2nd, Susanti Al, Maguire JD. Nephrotic syndrome and unrecognized Plasmodium malariae infection in a US Navy sailor 14 years after departing Nigeria. J Travel Med. 2011;18:288-91.

14. Guimaraes LO, Wunderlich G, Alves JM, Bueno MG, Rohe F, Catao-Dias JL, et al. Merozoite surface protein-1 genetic diversity in Plasmodium malariae and Plasmodium brasilianum from Brazil. BMC Infect Dis. 2015:15:529.

15. Muller HM, Reckmann I, Hollingdale MR, Bujard H, Robson KJ, Crisanti A. Thrombospondin related anonymous protein (TRAP) of Plasmodium falciparum binds specifically to sulfated glycoconjugates and to HepG2 hepatoma cells suggesting a role for this molecule in sporozoite invasion of hepatocytes. EMBO J. 1993;12:2881-9.

16. Robson KJ, Frevert U, Reckmann I, Cowan G, Beier J, Scragg IG, et al. Thrombospondin-related adhesive protein (TRAP) of Plasmodium falciparum: expression during sporozoite ontogeny and binding to human hepatocytes. EMBO J. 1995;14:3883-94.

17. Sultan AA, Thathy V, Frevert U, Robson KJ, Crisanti A, Nussenzweig V, et al. TRAP is necessary for gliding motility and infectivity of Plasmodium sporozoites. Cell. 1997;90:511-22.

18. Hodgson SH, Ewer KJ, Bliss CM, Edwards NJ, Rampling T, Anagnostou NA, et al. Evaluation of the efficacy of ChAd63-MVA vectored vaccines expressing circumsporozoite protein and ME-TRAP against controlled human malaria infection in malaria-naive individuals. J Infect Dis. 2015;211:1076-86.

19. Alexander DL, Mital J, Ward GE, Bradley P, Boothroyd JC. Identification of the moving junction complex of Toxoplasma gondii: a collaboration between distinct secretory organelles. PLoS Pathog. 2005;1:e17.

20. Alexander DL, Arastu-Kapur S, Dubremetz JF, Boothroyd JC. Plasmodium falciparum AMA1 binds a rhoptry neck protein homologous to TgRON4, a component of the moving junction in Toxoplasma gondii. Eukaryot Cell. 2006;5:1169-73.

21. Besteiro S, Michelin A, Poncet J, Dubremetz JF, Lebrun M. Export of a Toxoplasma gondii rhoptry neck protein complex at the host cell membrane to form the moving junction during invasion. PLoS Pathog. 2009;5:e1000309

22. Richard D, MacRaild CA, Riglar DT, Chan JA, Foley M, Baum J, et al. Interaction between Plasmodium falciparum apical membrane antigen 1 and the rhoptry neck protein complex defines a key step in the erythrocyte invasion process of malaria parasites. J Biol Chem. 2010;285:14815-22.

23. Lamarque M, Besteiro S, Papoin J, Roques M, Vulliez-Le Normand B, Morlon-Guyot J, et al. The RON2-AMA1 interaction is a critical step in moving junction-dependent invasion by apicomplexan parasites. PLoS Pathog. 2011;7:e1001276.

24. Tyler JS, Boothroyd JC. The C-terminus of Toxoplasma RON2 provides the crucial link between AMA1 and the host-associated invasion complex. PLoS Pathog. 2011;7:e1001282.
25. Thera MA, Doumbo OK, Coulibaly D, Laurens MB, Ouattara A, Kone AK, et al. A field trial to assess a blood-stage malaria vaccine. N Engl J Med. 2011;365:1004-13.

26. van Dijk MR, Janse CJ, Thompson J, Waters AP, Braks JA, Dodemont HJ, et al. A central role for P48/45 in malaria parasite male gamete fertility. Cell. 2001;104:153-64.

27. Arevalo-Herrera M, Vallejo AF, Rubiano K, Solarte Y, Marin C, Castellanos A, et al. Recombinant Pvs48/45 antigen expressed in E. coli generates antibodies that block malaria transmission in Anopheles albimanus mosquitoes. PLOS ONE. 2015;10:e0119335.

28. Barry AE, Schultz L, Buckee CO, Reeder JC. Contrasting population structures of the genes encoding ten leading vaccine-candidate antigens of the human malaria parasite, Plasmodium falciparum. PLoS ONE. 2009;: :e8497.

29. Chenet SM, Tapia LL, Escalante AA, Durand S, Lucas C, Bacon DJ. Genetic diversity and population structure of genes encoding vaccine candidate antigens of Plasmodium vivax. Malar J. 2012;11:68.

30. Vallejo AF, Martinez NL, Tobon A, Alger J, Lacerda MV, Kajava AV, et al. Global genetic diversity of the Plasmodium vivax transmission-blocking vaccine candidate Pvs48/45. Malar J. 2016:15:202.

31. Rutledge GG, Bohme U, Sanders M, Reid AJ, Cotton JA, Maiga-Ascofare $\mathrm{O}$, et al. Plasmodium malariae and P. ovale genomes provide insights into malaria parasite evolution. Nature. 2017;542:101-4.

32. Snounou G, Singh B. Nested PCR analysis of Plasmodium parasites. Methods Mol Med. 2002;72:189-203.

33. Snounou G, Viriyakosol S, Zhu XP, Jarra W, Pinheiro L, do Rosario VE, et al. High sensitivity of detection of human malaria parasites by the use of nested polymerase chain reaction. Mol Biochem Parasitol. 1993:61:315-20.

34. Librado P, Rozas J. DnaSP v5: a software for comprehensive analysis of DNA polymorphism data. Bioinformatics. 2009;25:1451-2.

35. Finn RD, Attwood TK, Babbitt PC, Bateman A, Bork P, Bridge AJ, et al. InterPro in 2017-beyond protein family and domain annotations. Nucleic Acids Res. 2017:45:D190-9.

36. Tamura K, Stecher G, Peterson D, Filipski A, Kumar S. MEGA6: molecular evolutionary genetics analysis version 6.0. Mol Biol Evol. 2013;30:2725-9.

37. Nei $M$, Gojobori T. Simple methods for estimating the numbers of synonymous and nonsynonymous nucleotide substitutions. Mol Biol Evol. 1986;3:418-26.

38. Tajima F. Simple methods for testing the molecular evolutionary clock hypothesis. Genetics. 1993;135:599-607.

39. Fu YX, Li WH. Statistical tests of neutrality of mutations. Genetics. 1993;133:693-709.

40. Chenet SM, Branch OH, Escalante AA, Lucas CM, Bacon DJ. Genetic diversity of vaccine candidate antigens in Plasmodium falciparum isolates from the Amazon basin of Peru. Malar J. 2008;7:93.

41. Babiker HA, Walliker D. Current views on the population structure of Plasmodium falciparum: implications for control. Parasitol Today. 1997:13:262-7.

\section{Submit your next manuscript to BioMed Central and we will help you at every step:}

- We accept pre-submission inquiries

- Our selector tool helps you to find the most relevant journal

- We provide round the clock customer support

- Convenient online submission

- Thorough peer review

- Inclusion in PubMed and all major indexing services

- Maximum visibility for your research

Submit your manuscript at www.biomedcentral.com/submit
BioMed Central 